\title{
ON THE CENTER OF SOME FINITE LINEAR GROUPS
}

\author{
HARVEY I. BLAU
}

ABSTRACT. This note proves two results, one in characteristic $p$ and the other in characteristic zero, which restrict the order of the center of some finite linear groups of degree less than a prime $p$ which divides the group order.

$G$ denotes a finite group, $p$ a fixed odd prime, $P$ a Sylow $p$-subgroup of $G . Z$ is the center of $G$ and $z=|Z|$.

Theorem 1. Assume $G=G^{\prime}, G$ is not of type $L_{2}(p), P$ is cyclic, and for some field $K$ of characteristic $p$, there is a faithful, indecomposable $K G$-module $L$ of dimension $d<p$. If $d$ is odd then $d \geq(z /(z+2)) p$. If $d$ is even then

$$
\begin{aligned}
d & \geq(z /(z+2))(p+1) & & (z \text { odd }) \\
& \geq(z /(z+4))(p+1) & & (z \text { even }) .
\end{aligned}
$$

Theorem 2. Assume $G=G^{\prime}, P$ has order $p$ and is not normal in $G$, the number $t$ of conjugate classes of p-elements of $G$ is at least 3 , and $G$ has a faithful irreducible complex character $\chi$ of degree $d<p-1$. Let $e=(p-1) / t$. Then $z \leq 2 d /(e+1)$.

Remarks. (i) Theorem 1 supplements [1, Theorem 5.11]. While the fractional multiples of $p$ given in [1, Theorem 5.11] are a little better than in Theorem 1, an annoying (especially for large values of $z$ ) remainder term in the earlier result is dispensed with here. One consequence is the following: It is known that $z \mid d$ under the hypotheses of Theorem 1. As a corollary of the theorem, we have that if $z=d$, then $d \geq p-3$.

(ii) Theorem 2 is proved by exploiting the methods of [2], which were themselves a variation on those of [8]. The numerical case $p=31, d=$ $z=28, e=3$ listed in $[1, \S 8]$, and not ruled out by previous results, is eliminated by Theorem 2. For in that case the modular representation involved in [1] lifts to an ordinary representation to which Theorem 2 can be applied.

Received by the editors September 3, 1974.

AMS (MOS) subject classifications (1970). Primary 20C15, 20C20; Secondary 20C05, $20 \mathrm{D} 05$.

Key words and phrases. Indecomposable modular representation, faithful irreducible complex representation, small degree, prime order Sylow subgroup, conjugate class of $p$-elements. 
(iii) Apparently, no groups are known which satisfy either the hypotheses of Theorem 1 with $p \geq 13$ and $d<p-2$, or the hypotheses of Theorem 2 with $p>7$ and $d<p-2$. If such groups do exist, their $p$-local structure is quite restricted, as our results indicate.

Proof of Theorem 1. Since $L_{P}$ is indecomposable [7], and remains indecomposable under all field extensions (as $P$ is cyclic), we may assume $K$ is a splitting field for all subgroups of $G$. Let $d=p-s$. By $[1,(5.3)]$, the nonprojective summands of $L \otimes L$ are $L_{i}, 0 \leq i \leq s-1$, of dimensions $2 i+1+m_{i} p$, with $\sum_{i=0}^{s-1} m_{i} \leq p-2 s$. Now $z \mid d[1$, Proposition 5.1] and by $[1,(5.10)]$,

$$
z \mid 2\left(2 i+1+m_{i} p\right), \quad 0 \leq i \leq s-1 .
$$

For any integer $i$ with $0 \leq i<(s-1) / 2$, let $i^{\prime}=s-1-i$. Then (1) implies

$$
z \mid 2\left(2 s+\left(m_{i}+m_{i}\right) p\right), \quad 0 \leq i<(s-1) / 2 .
$$

Suppose $d$ is odd. Then $z$ is odd, and (2) yields

$$
\left(m_{i}+m_{i^{\prime}}\right) p \equiv-2 s \quad(\bmod z), \quad 0 \leq i \leq s / 2-1 .
$$

Since $p \equiv s(\bmod z)$, and $(s, z)=1$, we have

$$
m_{i}+m_{i^{\prime}} \equiv-2 \quad(\operatorname{inod} z), \quad 0 \leq i \leq s / 2-1 .
$$

Thus $m_{i}+m_{i^{\prime}} \geq z-2,0 \leq i \leq s / 2-1$, so that

$$
p-2 s \geq \sum_{i=0}^{s-1} m_{i} \geq \sum_{i=0}^{s / 2-1}\left(m_{i}+m_{i^{\prime}}\right) \geq(s / 2)(z-2) .
$$

It follows that $s \leq(2 /(z+2)) p$, whence $d \geq(z /(z+2)) p$.

Suppose $d$ is even. If $z$ is odd, again we have

$$
m_{i}+m_{i^{\prime}} \equiv-2 \quad(\bmod z), \quad 0 \leq i<(s-1) / 2 .
$$

Also, $z \mid 2(s+p m(s-1) / 2)$ implies $m_{(s-1) / 2} \equiv-1(\bmod z)$. Thus

$$
p-2 s \geq m_{(s-1) i 2}+\sum_{i=0}^{(s-3) / 2}\left(m_{i}+m_{i^{\prime}}\right) \geq z-1+(z-2)(s-1) / 2 \text {. }
$$

It follows that $s \leq(2 /(z+2)) p-z /(z+2)$, hence

$$
d \geq(z /(z+2)) p+z /(z+2)=(z /(z+2))(p+1) .
$$

If $z$ is even, (2) implies the above congruences are still valid modulo $z / 2$. So we may replace $z$ by $z / 2$ in (3) to obtain $d \geq(z /(z+4))(p+1)$. 
Proof of Theorem 2. Assume the hypotheses of Theorem 2. Then $d=$ $p-e$ [5]. If $p=7$, no such groups exist [6], so we may assume $p>7$. Feit's reduction argument $[8,(6.1)]$ shows that $G$ is not of type $L_{2}(p)$. Then by $[8,(2.1)], G$ satisfies conditions $(*)$ of [8], and $z \mid d$. If $e=1$ then $d=p-1$, a contradiction. If $e=2$ then $G \approx \operatorname{SL}_{2}\left(2^{a}\right)$ and $z=1$ [9]. So we may assume $2<e<(p-1) / 2$. Also, [8, Theorem 1] implies $z$ is even, hence $e$ is odd.

Let $F$ be a $p$-adic number field with ring of integers $R$ such that $F$ and $R / J(R)=K$ are splitting fields for all subgroups of $G$. Let $M$ be an $R$-free $R G$-module such that $M \otimes_{R} F$ affords $\chi$ and $L=M / J(R) M$ is indecomposable [10]. $P \Varangle G$ implies $L$ is faithful, so the situation of Theorem 1 holds with $s=e$.

Let $N$ be the normalizer of $P$ in $G$, and let $L_{N}=V_{p-e}(\lambda)$ (cf. $[1, \S 5]$ ), where $\lambda$ is a linear character: $N \rightarrow K$. Let $L_{i}$ be as above, $0 \leq i \leq e-1$. $L_{i}$ has Green correspondent $V_{2 i+1}\left(\lambda^{2} \alpha^{e+i}\right)[1, \S 5]$. Let $\chi_{Z}=\chi(1) \eta, \eta$ a faithful linear character: $Z \rightarrow F$. Now $\eta(Z) \subseteq R$, and if $\bar{\eta}$ denotes $\eta$ composed with the canonical homomorphism: $R \rightarrow K$, then $\bar{\eta}=\lambda_{Z}$. There is a one-one correspondence between the p-blocks of positive defect and the distinct powers of $\eta$ : an irreducible character $\zeta$ of $G$ is in block $B_{n}$ if and only if $\zeta_{Z}=\zeta(1) \eta^{n}$ [4]. Thus $\chi, L$ are in $B_{1}$ and the $L_{i}$ are all in $B_{2}[1, \S 4]$.

Let $\zeta_{j}, 1 \leq j \leq t$, be the exceptional characters in $B_{2}$. Then $\zeta_{j_{Z}}=$ $\zeta_{j}(1) \eta^{2}$. By $[8,(4.1)], \zeta_{j}(1)=m p+e$ for some positive integer $m$, independent of $j$.

We may assume $F$ is sufficiently large so that for each $i$ with $0 \leq i<$ $(e-1) / 2$, there is an $R$-free $R G$-module $X_{i}$ such that $X_{i} / J(R) X_{i} \approx L_{i} \oplus$ $L_{e-i-1}[3$, Lemma 2.1]. Also, there is an $R$-free $R G$-module $Y$ such that $Y / J(R) Y \approx L_{(e-1) / 2}$. Now the $\zeta_{j}$ occur in the character afforded by each $X_{i}$ with total multiplicity at least 2 , and in the character afforded by $Y$ with multiplicity at least $1[3$, Lemma 2.2]. Hence

$$
\operatorname{dim}_{K} L \otimes L \geq \operatorname{rank}_{R}\left(Y \oplus \sum_{i=0}^{(e-3) / 2} X_{i}\right) \geq 2 \zeta_{1}(1)(e-1) / 2+\zeta_{1}(1)=e \zeta_{1}(1)
$$

Therefore $(p-e)^{2} \geq e(m p+e)$. It follows that $m \leq(p-2 e) / e=t-2+1 / e$, whence $m \leq t-2$.

Since $\zeta_{j_{Z}}=\zeta_{j}(1) \eta^{2}$, we set the determinant of the appropriate scalar matrices equal to 1 (as $G=G^{\prime}$ ) to see that $\eta^{2(m p+e)}=1$. Since $\eta$ is faithful on the cyclic group $Z$, it follows that $z \mid 2(m p+e)$, hence $m p+e \equiv 0$ $(\bmod z / 2)$. Now $z \mid p-e$ implies $m e+e \equiv 0(\bmod z / 2)$ and $(z, e)=1$ yields $m+1 \equiv 0(\bmod z / 2)$. Therefore $t-2 \geq m \geq z / 2-1$. Hence $z \leq 2 t-2=$ $2(d-1) / e<2 d / e$. Since $z \mid d$, we have $z \leq 2 d /(e+1)$. 


\section{REFERENCES}

1. II. I. Blau, Under the degree of some finite linear groups, Trans. Amer. Math. Soc. 155 (1971), 95-113; errata, ibid. 162 (1971), 475. MR 43 \#367; $44 \# 329$.

2. _- An in equality for complex linear groups of small degree, Proc. Amer. Math. Soc. 28 (1971), 405-408. MR 43 \#364.

3. - Finite groups where two small degrees are not too small, J. Algebra 28 (1974), 541-555.

4. R. Brauer, On groups whose order contains a prime number to the first power. I, Amer. J. Math. 64 (1942), 401-420. MR 4, 1.

5. - Some results on finite groups whose order contains a prime to the first power, Nagoya Math.J. 27 (1966), 381-399. MR 33 \#7402.

6. - Über endliche lineare Gruppen von Primzahlgrad, Math. Ann. 169 (1967), 73-96. MR 34 \#5913.

7. W. Feit, Groups with a cyclic Sylow subgroup, Nagoya Math. J. 27 (1966), 571-584. MR 33 \#7404.

8. —_ On finite linear groups, J. Algebra 5 (1967), 378-400. MR 34 \#7632.

9. _- On finite linear groups. II, J. Algebra 30 (1974), 496-506. \#7677.

10. J. G. Thompson, Vertices and sources, J. Algebra 6 (1967), 1-6. MR 34

DEPARTMENT OF MATHEMATICS, NORTHERN ILLINOIS UNIVERSITY, DE KALB, ILLINOIS 60115 